\title{
EXPERIMENTAL STUDY ON THE MEASUREMENT OF $S$ - $p$ RELATIONS OF LNAPL IN A POROUS MEDIUM
}

\author{
Masashi KAmoni), Yan Liii), Kazuto Endoiii), Toru Inuivi and TAKeshi Katsumivi)
}

\begin{abstract}
A simple and effective device for measuring saturation-capillary pressure relation ( $S$ - $p$ relation) in LNAPL-water system is designed with electrical conductivity probes, hydrophilic tensiometers and hydrophobic tensiometers. With this device, the $S$ - $p$ relation in LNAPL-water system is characterized. The $S$ - $p$ relation in air-LNAPL system is obtained by a scaling factor method. At a given saturation of wetting phase, it is found that the descending sequence of capillary pressures is in the order of air-water, LNAPL-water and air-LNAPL systems. The descending sequence of entry pressure and displacement pressure is also in the order of air-water, LNAPL-water and air-LNAPL systems. Furthermore, the relative permeability of air is larger than the LNAPL in their corresponding aqueous two phase systems, and the relative permeability of the LNAPL is slightly larger than water in their corresponding gaseous two phase systems.
\end{abstract}

Key words: capillary pressure, degree of saturation, electrical conductivity probe, light non-aqueous phase liquid (LNAPL), relative permeability, scaling factor method (IGC: B12/E7)

\section{INTRODUCTION}

Non-aqueous phase liquids (NAPLs) are hydrocarbon liquids and volatile organic compounds that are not readily miscible with water and air. Depending on their densities, NAPLs are typically classified as either light non-aqueous phase liquids (LNAPLs) that are lighter than water, or dense non-aqueous phase liquids (DNAPLs) that are denser than water.

When LNAPLs are released into the subsurface through spill or leak, they will migrate downward through the vadose zone. The fate, flow and transport of LNAPLs are governed by complex interactions among capillary, viscous and gravity force, mass transfer between phases and chemical and biological reactions. These processes are also affected by factors such as temperature, soil and fluid compressibility, soil heterogeneity, volume of spilled LNAPLs and geometry of the spill source. At a LNAPL polluted site, LNAPL forms the free lens above the water table or is trapped in the pores in the subsurface. Some evaporates into the air (volatilization), some sorbs the surface of the soil particles (sorption), and some dissolves into the groundwater (dissolved plume). Fluctuation of water table, which occurs due to pumping of groundwater in a large quantity or seasonal variety of rainfall, can also cause vertical movement and redistribution of mobile LNAPLs within the saturated and unsaturated zones. It is very important to determine the characteristics of LNAPLs migration in the subsurface with the fluctuation of water table in order to remediate the subsurface contamination effectively. In order to analyze and quantify the effects of water table fluctuation on the distribution of LNAPL in subsurface, there are mainly three methods. One is an analytic method, in which, the in-situ complicated problem is simplified, and thus the analytic solution of the in-situ problem is obtained, with errors to some extent. One is a physical simulation, on which, a prototype system (i.e., the in-situ complicated system) is simulated with a model-based system, i.e., an ex-situ device. The ex-situ device is a reductive in-situ system, which is obtained by reducing the in-situ system to a scale as requested by the theory of similarity. Another is a numerical simulation. With regard to this method, the relationship between the water saturation and the capillary pressure ( $S-p$ relation) is needed. To measure the capillary pressure as a function of water saturation, five main methods are known, i.e., long column, centrifuge, vapor pressure, pressure cell and Brooks' method. Each of them has innumerable variations (Corey, 1994); however, the measurement of water saturation in a porous medium is a major task during meas-

i) Professor, Graduate School of Global Environmental Studies, Kyoto University, Japan.

ii) Graduate Student, ditto (Currently Lecturer, Sun Yat-sen University, China).

iii) Researcher, Research Center for Material Cycles and Waste Management, National Institute for Environmental Studies, Japan.

iv) Assistant Professor, Graduate School of Global Environmental Studies, Kyoto University, Japan (inui@mbox.kudpc.kyoto-u.ac.jp).

v) Associate Professor, ditto.

The manuscript for this paper was received for review on September 24, 2004; approved on October 2, 2006.

Written discussions on this paper should be submitted before September 1, 2007 to the Japanese Geotechnical Society, 4-38-2, Sengoku, Bunkyo-ku, Tokyo 112-0011, Japan. Upon request the closing date may be extended one month. 
urement of $S$ - $p$ relations. Although gravimetric sampling method for measuring water saturation is the most accurate method, it can not provide timely and accurate water saturation continuously and automatically, because soil samples must be removed from a soil mass. In laboratory experiments, generally, the non-intrusive or non-destructive methods should be used to measure fluid saturation. Widely accepted in situ methods for measuring soil water saturation are radioactive methods such as the neutron scattering method (Gardner and Kirkham, 1951), X-ray or the gamma ray attenuation method (Host-Madsen and Jensen, 1992; Illangasekare et al., 1995a, 1995b; Lenhard et al., 1993; Reginato and van Bavel, 1964; Tidwell and Glass, 1994), Time Domain Reflectometry (TDR) method (Topp et al., 1980), and so on. These methods are quite accurate and non-destructive; however, they require special caution to avoid possible health hazards or higher investment. In this paper, water saturation is measured with electrical conductivity probes (Endo, 2002; Kamon et al., 2003).

Migration of a fluid in a saturated porous medium is expressed by Darcy's law, which can also be extended to describe a multiphase flow (Bear, 1979; Sharma, 2000). In a multiphase flow system, flux of each fluid is a function of its effective saturation (Brooks and Corey, 1964). The unsaturated hydraulic conductivity, which is linked with the saturation and capillary pressure, should be determined to simulate accurately a LNAPL distribution in an unsaturated porous medium. However, the relationships among capillary pressure, saturation and unsaturated hydraulic conductivity are different during drying and wetting processes because of contact angle hysteresis and nonwetting fluid entrapment. Due to the practical difficulties encountered in measuring the unsaturated hydraulic conductivity, considerable efforts have been made to estimate the value of unsaturated hydraulic conductivity from the relationships between capillary pressure and saturation (Lenhard and Parker, 1987a).

Furthermore, obtaining the relationship between saturation and capillary pressure for a different phase in a three-phase system (air-LNAPL-Water) is time consuming and no standard procedure is available for simultaneous measurements of the saturation and pressure of water and LNAPL in a porous medium. Only a few results were presented so far for the migration of LNAPLs in unsaturated sand using large-scale tanks (Kechavarzi et al., 2000). Therefore, researchers have suggested the use of $S$ - $p$ relations in two-phase system to predict those in three-phase system (Parker et al., 1987). Lenhard and Parker (1988) developed an experimental apparatus to measure directly the relationships between saturation and capillary pressure in a three-phase system with unconsolidated porous media. Their results indicated a close agreement between water saturation in a three-phase system and that in a two-phase system as a function of water-LNAPL capillary pressure.

In this paper, there are two features that are being focused on. One is to measure the $S$ - $p$ relations with the electrical conductivity probes in LNAPL-water system. The other is to obtain the characteristics of LNAPL migration in a porous medium to simulate accurately an LNAPL distribution in subsurface.

\section{CONSTITUTIVE MODELING}

\section{Capillary Pressure Head}

In a two-phase porous medium system for monotonic displacement of a wetting fluid $j$ by a nonwetting fluid $i$, the capillary pressure $P_{\mathrm{ij}}$ can be given as:

$$
P_{\mathrm{ij}}=P_{\mathrm{i}}-P_{\mathrm{j}}
$$

where $P_{\mathrm{i}}$ is nonwetting phase pressure; and $P_{\mathrm{j}}$ is wetting phase pressure.

Instead of pressures, it is convenient to employ capillary head, $h_{\text {cij }}$, which is defined on an equivalent water height basis by:

$$
h_{\mathrm{cij}}=P_{\mathrm{ij}} / \rho_{\mathrm{w}} g
$$

where $\rho_{\mathrm{w}}$ is the density of water; and $g$ is the scalar magnitude of gravitational acceleration.

In the case of LNAPL and water two-phase system, two kinds of probes for measuring the pore liquid pressures are required. One is hydrophilic tensiometer with water-wetting ceramic cup to measure pore water pressure and the other is a hydrophobic tensiometer with a LNAPL-wetting ceramic cup to measure pore LNAPL pressure. The capillary pressure is simply the difference between the higher one and the lower one of the two pressure readings (Dullien, 1992).

\section{$k$-S-p Relations}

The relationship between saturation of phase $j, S_{\mathrm{j}}$, and capillary pressure head $h_{\mathrm{c}}$ in unsaturated porous media with immiscible fluid phases, pair $i$ and $j$, is referred to as a water retention curve or $S$ - $p$ relation, where $i$ and $j$ stand for nonwetting phase and wetting phase respectively. A widely accepted empirical parameter form given by van Genuchten (1980) was written as (Helmig, 1997):

$$
h_{\mathrm{cij}}=\frac{1}{\alpha}\left(S_{\mathrm{je}}^{-1 / m}-1\right)^{1 / n} \quad h_{\mathrm{cij}}>0
$$

Equation (3) refers to van Genuchten model (VG model), where $\alpha(1 / \mathrm{cm})$ and $n$ are parameters, and $m=1-1 / n$.

The effective saturation of the $j$ phase, $S_{\mathrm{je}}$ is defined as:

$$
S_{\mathrm{je}}=\frac{S_{\mathrm{j}}-S_{\mathrm{jr}}}{1-S_{\mathrm{jr}}}
$$

where $S_{\mathrm{jr}}$ is the residual or the irreducible saturation of the wetting phase $j$.

Another famous empirical form was given by Brooks and Corey (1964) (BC model), and the equation was written as:

$$
h_{\mathrm{cij}}=h_{\mathrm{d}} S_{\mathrm{je}}^{-1 / \lambda} \quad h_{\mathrm{cij}}>h_{\mathrm{d}}
$$

where $\lambda$ indicates the pore distribution index and $h_{\mathrm{d}}$ is the displacement pressure head of the reference two-phase system, that is, the displacement pressure head from the 
phase $j$ to the other phase $i$.

In a porous medium with multiphase flows, Darcy's Law is extended as follows:

$$
v_{\mathrm{i}}=-\left(k_{\mathrm{ei}} / \mu_{\mathrm{i}}\right)\left(\nabla P_{\mathrm{i}}-\rho_{\mathrm{i}} g\right)
$$

where $v_{\mathrm{i}}$ is defined as average velocity of fluid $i ; P_{\mathrm{i}}$ is hydrostatic pressure of fluid $i ; \mu_{\mathrm{i}}$ is viscosity of fluid $i$; $\rho_{\mathrm{i}}$ and $k_{\text {ei }}$ are density and effective permeability respectively.

The effective permeability, $k_{\mathrm{e}}$, is generally expressed as a function of the intrinsic permeability $k$ of the medium, which is defined as follows:

$$
k_{\mathrm{ei}}=k k_{\mathrm{ri}}
$$

where $k_{\text {ri }}$ is called relative permeability of fluid $i$.

The method for predicting relative permeability is also proposed as the VG and BC models, and the value of the relative permeability is estimated from the parameters of the $S$ - $p$ relations fitted with VG and BC models.

As for VG model, the relative permeability of the nonwetting phase, $k_{\mathrm{ri}}$, and that of wetting phase, $k_{\mathrm{rj}}$, can be described as follows:

$$
\begin{aligned}
& k_{\mathrm{rj}}=S_{\mathrm{je}}^{\varepsilon}\left[1-\left(1-S_{\mathrm{je}}^{1 / m}\right)^{m}\right]^{2} \\
& k_{\mathrm{ri}}=\left(1-S_{\mathrm{je}}\right)^{\gamma}\left[1-S_{\mathrm{je}}^{1 / m}\right]^{2 m}
\end{aligned}
$$

where $\varepsilon$ and $\gamma$ representing the conductivity of pore structure can be regarded as $\varepsilon=1 / 2$ and $\gamma=1 / 3$, respectively (Helmig, 1997).

As for BC model, the values of them can be expressed as follows.

$$
\begin{aligned}
& k_{\mathrm{rj}}=S_{\mathrm{je}}^{(2+3 \lambda) / \lambda} \\
& k_{\mathrm{ri}}=\left(1-S_{\mathrm{je}}\right)^{2}\left(1-S_{\mathrm{je}}^{(2+\lambda) / \lambda}\right)
\end{aligned}
$$

\section{MATERIALS AND EXPERIMENT}

\section{Experimental Apparatus}

The schematic for measuring $S$ - $p$ relations in LNAPLwater porous medium system is shown in Fig. 1. An acrylic column $(35 \mathrm{~mm}$ inner diameter, $45 \mathrm{~mm}$ outer diameter, and $50 \mathrm{~mm}$ in length) was utilized, with two pairs of tensiometers assembled along one side, and three electrical conductivity probes assembled on the opposite side. A pair of tensiometers is composed of a hydrophobic tensiometer and a hydrophilic tensiometer for measuring the pore LNAPL pressure and the pore water pressure respectively. At the same position of a pair of tensiometers, an electrical conductivity probe was utilized to measure water saturation. Only the pore liquid pressure, $P_{\mathrm{i}}$ and $P_{\mathrm{j}}$, and water saturation data measured at the same position were adopted for $S-p$ relation description. A rotary pump was utilized to pump the pore liquid out of the column.

A testing program is composed of the drainage process and the wetting process. During drainage process, firstly, sand sample was fully saturated with water. Then pore water was pumped out of the column at the bottom. Simultaneously, same volume of the LNAPL was infiltrated into the sand sample from the top of the column.

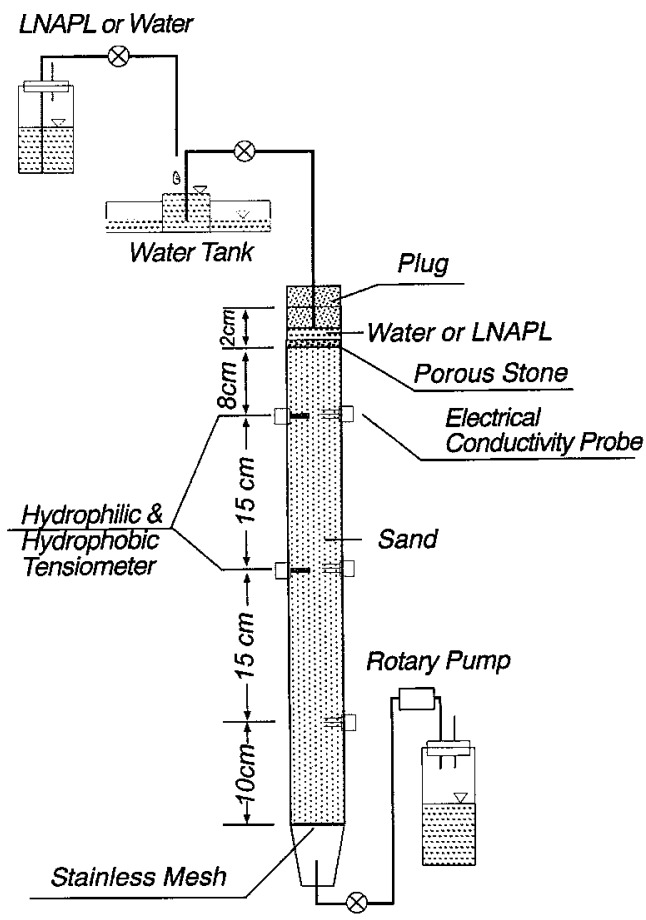

Fig. 1. Experimental apparatus for measuring $S-p$ relations in a LNAPL-water system

The drainage process was continued until LNAPL began to be pumped out from the bottom. After drainage process had completed, the wetting process began. The LNAPL was pumped out of the column from the bottom, and same volume of water was infiltrated into the sand sample from the top of the column simultaneously. The wetting process was completed when water began to be pumped out of the column. During the drainage or the wetting process, there was always a liquid layer above the sand sample in the column to prevent air from penetrating into the sand sample. It can be assured that the tests were always in a LNAPL-water system during both the drainage and wetting processes.

\section{Materials and Samples Preparation}

Toyoura sand, with a particle density of $2.64 \mathrm{~g} / \mathrm{cm}^{3}$, compacted-state void ratio of 0.62 , and saturated density of $2.01 \mathrm{~g} / \mathrm{cm}^{3}$, was used as a sandy porous medium for the column test. The grain size distribution of Toyoura sand is shown in Fig. 2.

A sodium chloride solution with the concentration of $0.05 \mathrm{~mol} / 1$ was used as the initial pore water to raise the reactivity of the electrical conductivity probe and to avoid the formation of condenser component in the sand system (Kamon et al., 2003). The full water saturation of sandy medium was achieved by an underwater falling method. The sand sample was poured into the column via a funnel in uniform layers. Then, the pore water was induced from the bottom of the column, and the corresponding volume of the sodium chloride solution was filled into the sand medium from the top of the column. Thus, before the drainage process began, the sandy medium had been fully saturated with the sodium 
chloride solution. Paraffin liquid was used as a substitute for LNAPL, due to its very low volatility at room temperature, negligible solubility in water and safety to health. In order to distinguish visually the downward migration of the interface between the LNAPL and the water during the drainage process, LNAPL was dyed with Sudan III with the weight ratio of 10000:1. The properties of materials used in this test are shown in Table 1.

\section{WATER SATURATION MEASUREMENT}

\section{Electrical Conductivity Probe}

Conductance (reciprocal of resistivity) of sands is mostly due to the presence of water, and electrical resistivity is a function of water content since it is the major conductive material of the soil matrix. In a multiphase system composed of NAPL, air, water, and pure sand, water will be the principal conductor of electrical current while air, sand particles, and NAPL, which have a larger resistance, will act as insulators. Thus soil system with higher water saturation is endued with a lower electrical resistivity. The water saturation in a porous medium can be measured by the changes in electrical resistivity of this medium as described by Archie $(1942,1947)$. Since then, a four-electrode probe has been the standard for the electrical investigation of soil system in both fields and

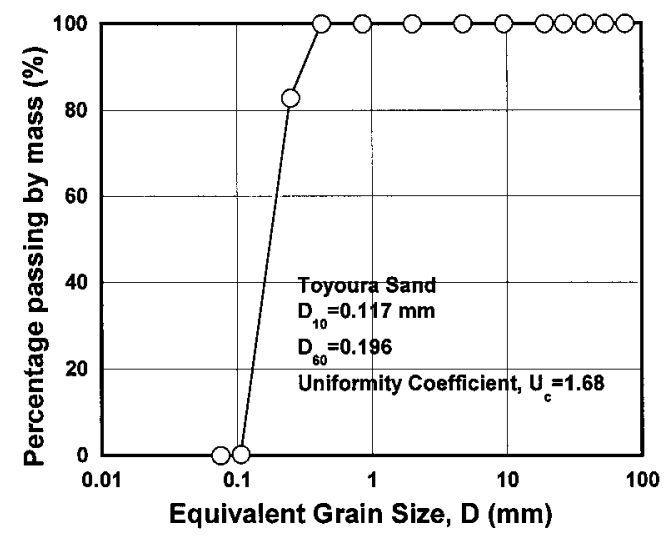

Fig. 2. Grain size distribution of Toyoura sand laboratories. Due to the difficulty of designing a multichannel system and several seconds needed for measuring one signal for a four-electrode probe, in this paper threeelectrode electrical conductivity probes were utilized to estimate the water saturation via the measurement of the electrical conductivity of sand system under immiscible two-phase flow condition. Although this electrical conductivity probe was originally developed to measure DNAPL saturation in a porous medium, it can also be used to measure the saturation of LNAPL, which also acts as an insulator in the LNAPL-water system.

Compared to FDR and TDR, the electrical conductivity probe designed by Endo (2002) is very cheap and easy to make by oneself. It is feasible for a column test due to its small size. The electrode of the electrical conductivity, whose diameter is about $1.0 \mathrm{~mm}$ and length is about 15 $\mathrm{mm}$, is almost non-destructive to the sands. Each of them has three parallel electrodes. Thus, there is no interference between two adjacent probes. The disadvantages of the electrical conductivity probe are also obvious. The measuring accuracy of this electrical probe for water saturation is slightly low. The electrical conductivity probe is only feasible for the porous media, whose pore water is electrolyte. When this electrical conductivity probe is utilized for the measurement of water saturation, the initial voltage of the resistance attached to it is necessary. Thus this three-electrode electrical conductivity probe is not feasible for in-situ situation.

\section{Calibration of Electrical Conductivity Probes}

Prior to the calibration, it was confirmed that the conductivity values of the LNAPL, air, and sand were negligible compared to that of the $0.05 \mathrm{~mol} / 1$ sodium chloride solution by measuring the voltage of the resistance attached to the probe inserted in the pure LNAPL, and dry sand samples.

For measuring the water saturation, power source of $1.0 V_{\text {pp }}$ (peak to peak voltage of AC) in output voltage and frequency of $0.1 \mathrm{~Hz}$, provided by a Function Synthesizer, were selected. Low voltage of AC was used so that probes could not interfere with one another, and electrolytic reactions in the vicinity of the electrodes could not happen either. Data recorded by a data logger

Table 1. Properties of materials used for measuring $S$-p relations in LNAPL-water system

\begin{tabular}{|c|c|c|c|}
\hline & Paraffin liquid & Sudan III & Water \\
\hline Formula & - & $\mathrm{C}_{22} \mathrm{H}_{16} \mathrm{~N}_{4} \mathrm{O}$ & $\mathrm{H}_{2} \mathrm{O}$ \\
\hline Appearance & Colorless, odorless & Red powder & Colorless \\
\hline Melting point & $<-10^{\circ} \mathrm{C}$ & $100^{\circ} \mathrm{C}$ & $0^{\circ} \mathrm{C}$ \\
\hline Evaporation rate & Nonvolatile & - & - \\
\hline Specific gravity & 0.87 & - & 1.00 \\
\hline Water solubility & Insoluble & $<0.1 \mathrm{~g} / 1$ & - \\
\hline Viscosity $(\mathrm{mPa} \cdot \mathrm{s})$ & $170.0\left(20^{\circ} \mathrm{C}\right)$ & - & - \\
\hline Hazard nature & Non toxic & - & - \\
\hline Surface tension $(\mathrm{mN} / \mathrm{m})$ & $31.07\left(25^{\circ} \mathrm{C}\right)$ & - & $72.75\left(20^{\circ} \mathrm{C}\right)$ \\
\hline Interfacial tension $(\mathrm{mN} / \mathrm{m})^{1)}$ & $62.06\left(25^{\circ} \mathrm{C}\right)$ & - & - \\
\hline Electrical conductivity $(\mathrm{mS} / \mathrm{cm})$ & 0.0 & - & - \\
\hline
\end{tabular}

1) Measured by Ring method (Huh and Mason, 1975) 


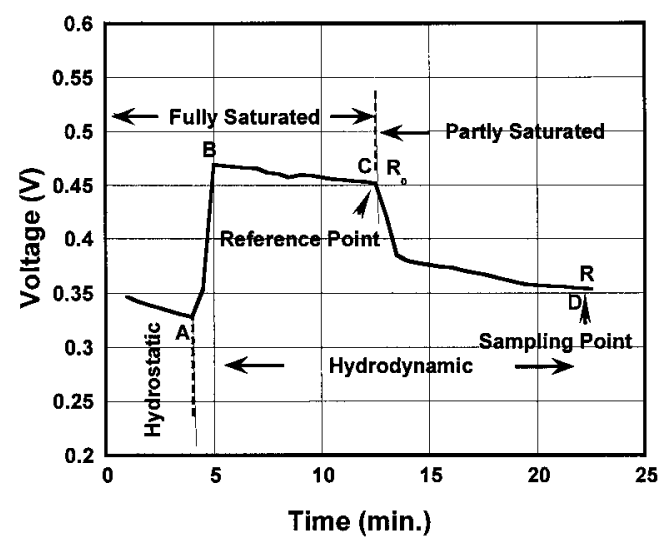

Fig. 3. Variety of output voltage during a drainage process

was only the output voltages across the resistance attached to the electrical conductivity probe. In order to estimate the water saturation of the sand sample, it is necessary to get a relationship between output signals and water saturations. Generally, this kind of probe needs to be calibrated for every porous medium sample.

According to the Ohm's law, if the resistance across the sand sample has a good relation with its water saturation, the voltage across the same sample must have a good relation with its water saturation. Thus the output voltage across the resistance attached to the probe could be utilized as an important parameter to estimate the water saturation.

The output voltages across the resistance attached to the probe, recorded by the data logger with a sample period of 0.5 second, are of sine wave. Cosine function, based on the fitting method by Chapra and Canale (1998), was selected to fit the data to obtain the amplitudes of the output signals.

The variety of output voltages across a resistance attached to an electrical conductivity probe in a porous medium during a whole drainage process is shown in Fig. 3. Until point $A$, the sand sample is under a hydrostatic condition, and fully saturated with the sodium chloride solution. The output voltage firstly decreases slowly to achieve its equilibrium, due to charging of the sand sample (Iitani and Masuda, 1975). When water begins to be pumped out of the column, the water flow increases the conductivity of the porous medium. Thus the voltage of the resistance attached to the probe increases suddenly to point $\mathrm{B}$. Then it maintains a constant value or decreases slightly to point $C$, which is the output voltage value when the water table fell down just above the electrical conductivity probe. With regard to the line $\mathrm{B}$ to $\mathrm{C}$, it is normally parallel, and the voltage at any point on the line is almost constant. However, if the charging process has not reached its balance at point $\mathrm{A}$ and the voltage of point $\mathrm{A}$ has not got its stable value, the line $\mathrm{B}$ to $\mathrm{C}$ will slightly decreases due to the further charging process. Accordingly, after the sample preparation is finished, and before the drainage process begins, the sand sample fully saturated with water should be placed for several hours under a hydrostatic condition. Only when

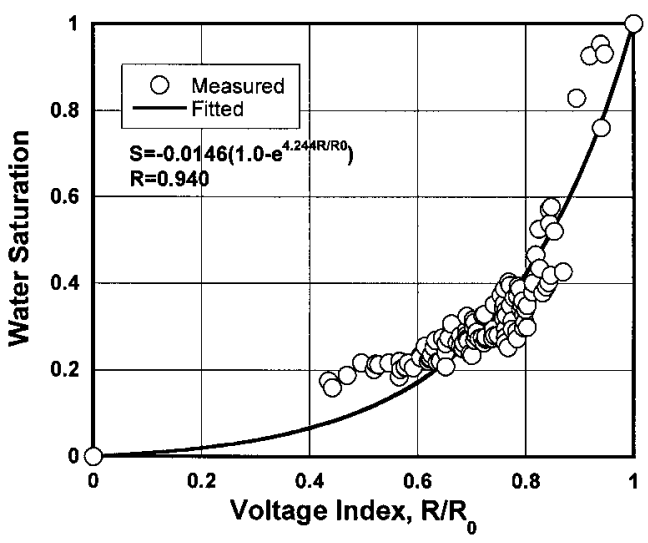

Fig. 4. Output voltage index-water saturation relation in air-water system

the charging process has gotten its balance then the drainage process begins.

Till point $\mathrm{C}$, the sand sample around the electrical conductivity probe is still fully saturated with water. After the water table passes through the electrical conductivity probe, the output voltage decreases with the reduction of water saturation of the sand sample nearby the electrical conductivity probe. At point $\mathrm{D}$, pump and data logger were stopped, and the sand sample around the probe was obtained from the column for measuring water saturation with a gravimetric method. During this drainage process, the output voltage at point $\mathrm{C}, R_{0}(\mathrm{~V})$ is taken as a reference value. Namely, water saturation equals to 1 if the output voltage, $R(\mathrm{~V})$, recorded by the data logger is equal to or bigger than $R_{0}$ under a hydrodynamic condition. It is also tested that the water saturation is zero when $R$ is equal to zero.

\section{Air-Water System}

In order to obtain the sand sample with its water saturation known and its corresponding output voltage value across the attached resistance, the drainage process was imposed by a rotary pump to extract pore water out of the column from its bottom. When there was no water to flow out of the column, the sand sample located around the probe was extracted as a specimen for measuring water saturation with gravimetric method. Its corresponding output voltage value across the attached resistance was also recorded with a data logger. This procedure was conducted with different draining rates or draining periods to get sand samples with different water saturations. Figure 4 shows the relation between output voltage, and water saturation under different water flow conditions. From Fig. 4, a good relationship between $S_{\mathrm{w}}$ and $R / R_{0}$ is observed and expressed with the empirical equation shown in Eq. (12). This indicates that a relationship between $S_{\mathrm{w}}$ and $R / R_{0}$ is not significantly affected by the water flow condition.

$$
S_{\mathrm{w}}=A\left(1.0-e^{B R / R_{0}}\right)
$$

where $S_{\mathrm{w}}$ is water saturation; and $A$ and $B$ are fitting parameters respectively. 


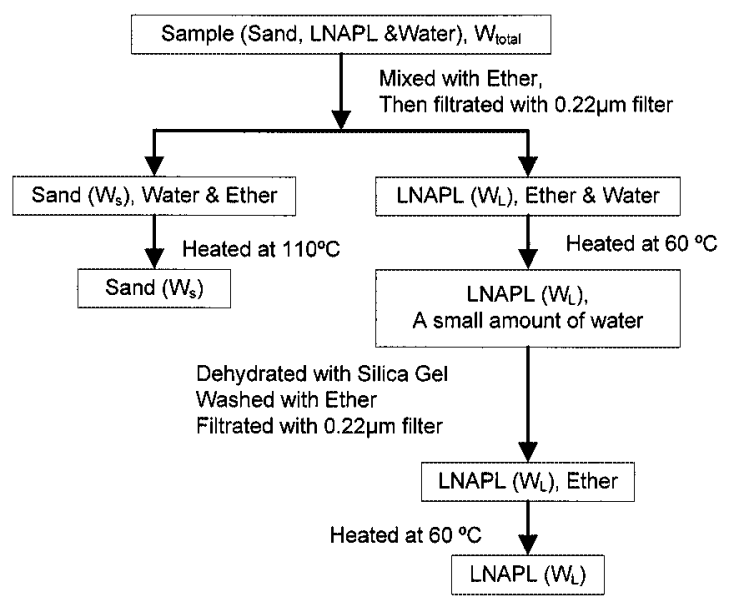

Fig. 5. Method for measuring the water content of a sand sample containing both LNAPL and water

\section{LNAPL-Water System}

In LNAPL-water system, it was found that, to control the water saturations of the sand samples by using different draining rates and draining periods in the calibration of these electrical conductivity probes was not effective. After LNAPL-water interface passes a fully saturated sand sample, most of the pore water is compelled out of the sample, and the water saturation of the sand sample is nearly constant. It is more effective to obtain sand samples with different water saturations by controlling the LNAPL-water interface position. After the sand sample was removed from the column, the normal gravimetric method could not be utilized to measure the water saturation, because the sand sample was composed of pure sand, LNAPL and water. Thus a method shown in Fig. 5 was designed to separate sand, LNAPL and water out of the sand sample.

In order to testify the feasibility of this separation method, two blank tests were conducted. For the test sample preparation, sand, water and LNAPL of known weights were mixed together and kept for 24 hours. The average error between the water saturations determined by this method and the real ones is only $1.66 \%$.

The $S_{\mathrm{w}}-R / R_{0}$ relation in LNAPL-water system is shown in Fig. 6. On the basis of Figs. 4 and 6, it can be found that the $S_{\mathrm{w}}-R / R_{0}$ curves in air-water and in LNAPL-water systems are similar. The difference is that the curvature in air-water system is somewhat bigger, although the conductivity of the LNAPL and the air is negligible compared to that of the sodium chloride solution. This would be caused by the wettability of the gilded electrode of the electrical conductivity probe (Kamon et al., 2003). Assuming that the wettability of the electrode is water-wet in the water-air system, and the pore air having no excess pressure, the pore water forms water film around the electrode, and the contact angle between water and the electrode becomes less than $90^{\circ}$. In the water-LNAPL system, when the pore LNAPL invades into the water-saturated pore space with the pressure higher than the pore water pressure and hits to a surface

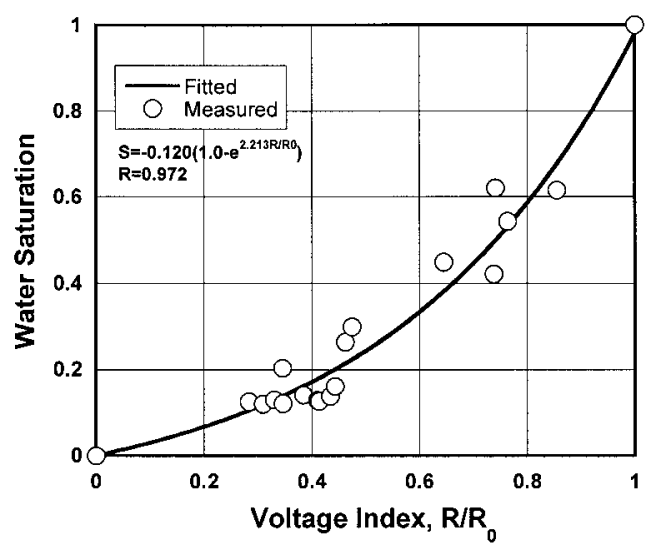

Fig. 6. Output voltage index-water saturation relation in LNAPLwater system

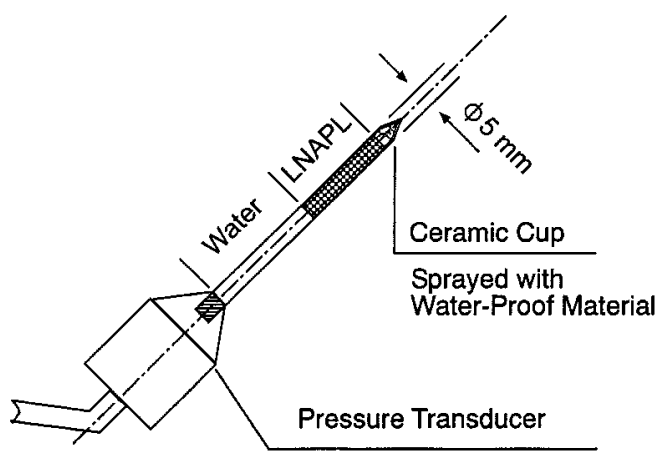

Fig. 7. Schematic of modifying a hydrophilic tensiometer to be a hydrophobic one

of the electrode, the pore water may not form water film around the electrode. In addition, there would be a possibility to change the wettability of the electrode from water-wet to oil-wet.

\section{Capillary Pressure Measurement}

Tensiometers (SWT5, Delta-T Devices Ltd.) were utilized to measure pore liquid pressure. Generally, a tensiometer is designed to measure the pore water pressure, and a hydrophobic tensiometer suitable for measuring the LNAPL pressure, was not available. Thus, a ceramic cup of a normal tensiometer should be modified to be hydrophobic. There are some modification methods proposed, one is spraying water-proof material to the ceramic cup (Endo, 2002; Kamon et al., 2003) and the other is soaking the ceramic cup in a Glassclad 18 solution for 20 minutes, then oven-drying it at $100^{\circ} \mathrm{C}$ for 1.5 hours (Busby et al., 1994). The former method was selected in the experiment. The schematic of a hydrophobic tensiometer is shown in Fig. 7. The hydrophobic tensiometer consists of a porous cup sprayed with the waterproof material, saturated with LNAPL, and a shaft filled with LNAPL and de-aired water for the prevention of LNAPL volatilization from the screw-type connection between the shaft and the pressure transducer, and the stabilization of the output signal from the pressure transducer. 


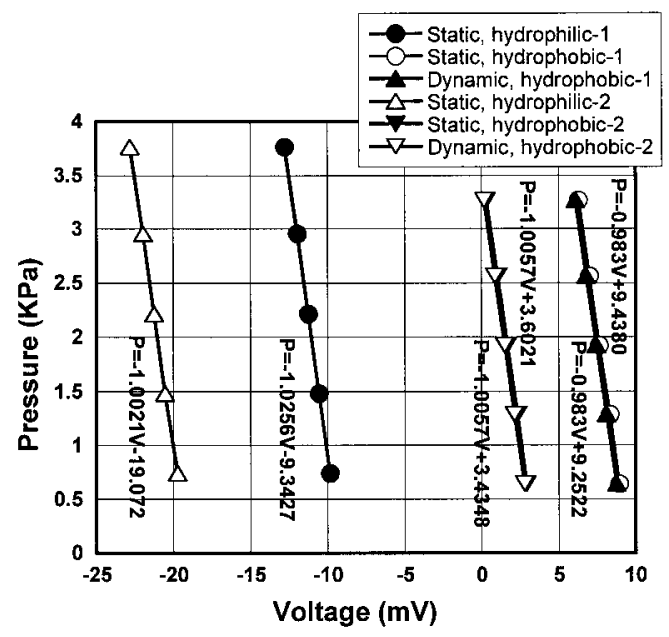

Fig. 8. Calibration results of hydrophilic and hydrophobic tensiometers

During measurement of pore liquid pressure, the signal from tensiometer recorded by the data logger was output voltage. A relation between voltage and pressure could be obtained in the calibration test (Fig. 8). The tensiometer is calibrated under a positive pressure condition. It is very difficult to calibrate a tensiometer with ceramic cup fully saturated with water or LNAPL under a negative pressure condition, because it is very difficult to measure the variety of negative pressure of the water or LNAPL in the ceramic cup of a tensiometer with present testing techniques.

In this column test, four tensiometers were used together with one power supply. The output voltages of tensiometers are recorded by way of a data logger. However, the output signals from these tensiometers will interfere with each other due to the limitation of the data logger, which is a single end data logger. Thus the isolation amplifier (Endo, 2002) was utilized between these tensiometers and the data logger. It resulted in the voltage of zero pressure of every tensiometer being different. However their slopes of relation between the voltage and the pressure are almost the same. If the isolation amplifier is not used, the output voltage of every tensiometer at zero pressure is zero.

When liquid was filled in the column without sand at different liquid levels, the hydrostatic or LNAPL-static pressures and their corresponding output voltages from hydrophilic or hydrophobic tensiometers were measured. It was also found that when liquid was pumped out of the column (hydrodynamic or LNAPL-dynamic condition), the voltage from a tensiometer is slightly different from that in a liquid-static condition even at the same liquid level. Figure 8 indicates that the correlation coefficients range from 0.9997 to 0.9999 . Voltage-pressure relations of hydrophilic tensiometers in hydrostatic condition and those of hydrophobic tensiometers in LNAPL-dynamic condition were selected for $S$ - $p$ relation measurement.

Although we can not calibrate under a negative liquid pressure condition, we could calibrate the pressure transducer of a tensiometer under a negative air pressure

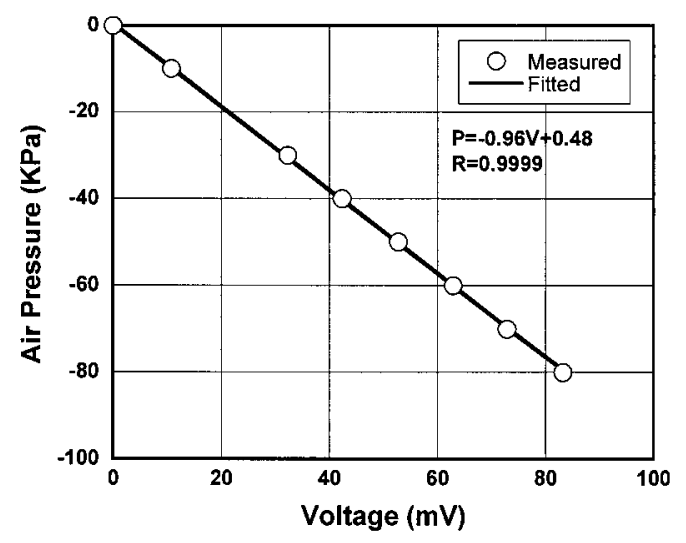

Fig. 9. Calibration result of the tensiometer under negative air pressures

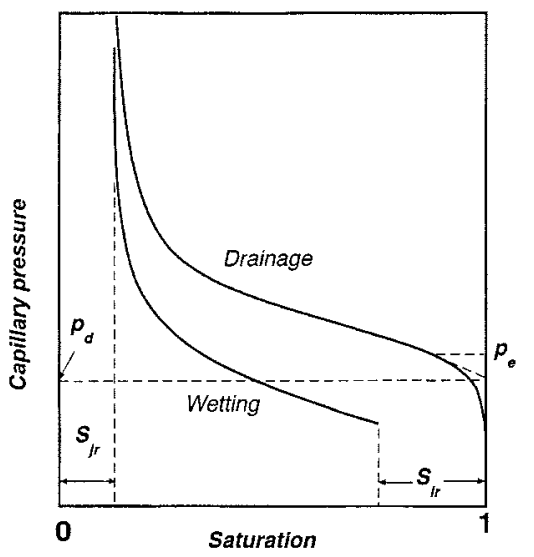

Fig. 10. Definition of $p_{\mathrm{e}}$ and $p_{\mathrm{d}}$ during drainage and wetting processes (Corey, 1994)

condition. The calibration result is shown in Fig. 9. It is found that the slope of the voltage-pressure relation is 0.96 , and it is very close to those of tensiometers calibrated with the positive pressure. Moreover, the relation between pressure and output voltage is linear. From these observations, it is acceptable that the negative pressure is extrapolated based on the voltage-pressure relation shown in Fig. 8.

\section{RESULTS AND DISCUSSIONS}

S-p Relations in Air-Water and LNAPL-Water Systems

The displacement pressure head, $p_{\mathrm{d}}$, is the capillary pressure at which the first desaturation on a drainage cycle occurs. At a pore space initially fully occupied with water, a finite value of capillary pressure, designed as $p_{\mathrm{e}}$, must be exceeded before nonwetting fluid can intrude into this element of the pore volume. The value of $p_{\mathrm{e}}$ is called entry pressure. The definition of $p_{\mathrm{e}}$ and $p_{\mathrm{d}}$ is shown in Fig. 10. $p_{\mathrm{d}}$ is somewhat smaller than $p_{\mathrm{e}}$ (Corey, 1994).

The measured $S$ - $p$ relations in the LNAPL-water system under the drainage/wetting process are shown in Fig. 11, where the one in the air-water system under the drainage process (Kamon et al., 2003) is also plotted. These $S$ - $p$ relations are obtained at the same test condi- 


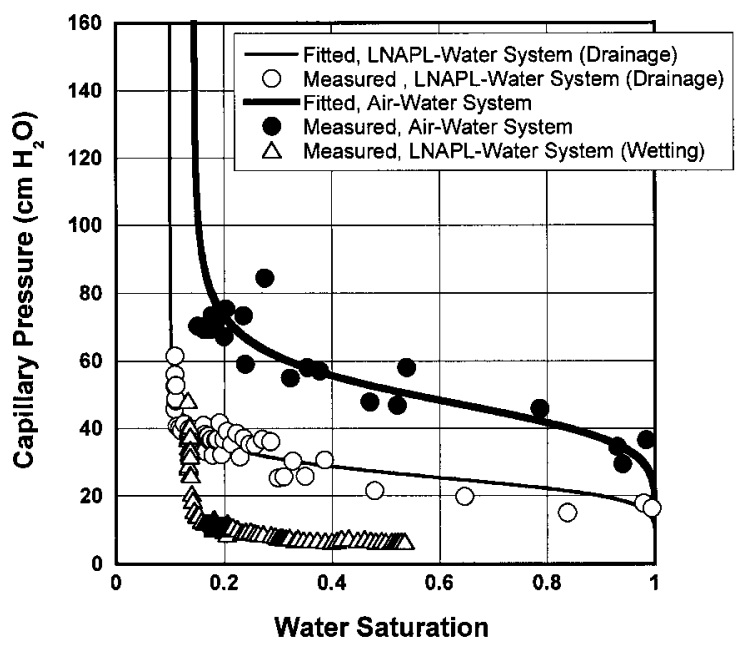

(a)

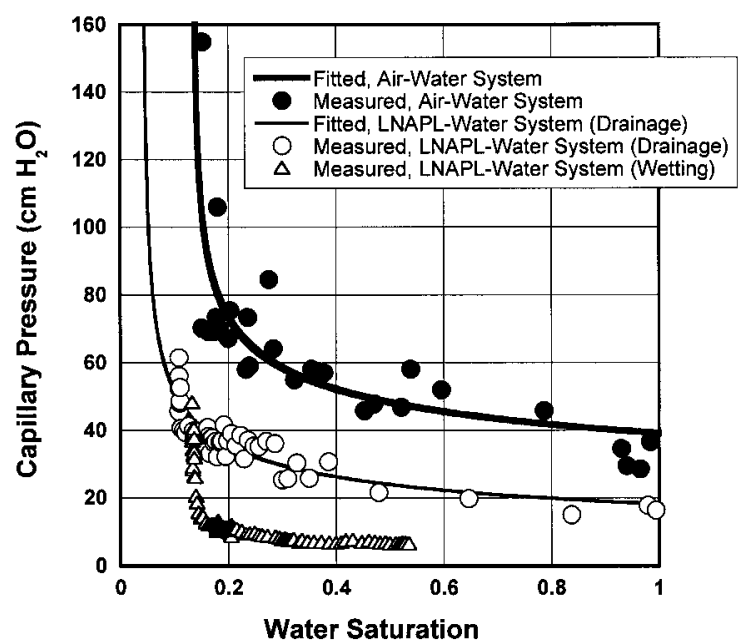

(b)

Fig. 11. Measured $S$ - $p$ relations in Toyoura sand: (a) Fitted with VG model and (b) Fitted with BC model

Table 2. Parameters of $S$ - $p$ relations fitted with VG and BC models in two-phase systems

\begin{tabular}{|c|c|c|c|c|c|c|c|c|}
\hline & \multicolumn{4}{|c|}{ VG model } & \multicolumn{4}{|c|}{ BC model } \\
\hline & $A$ & $n$ & $S_{\mathrm{r}}$ & $R^{1)}$ & $h_{\mathrm{d}}$ & $\lambda$ & $S_{\mathrm{r}}$ & $R^{1)}$ \\
\hline Air-water & 0.021 & 7.37 & 0.15 & 0.78 & 39.21 & 4.15 & 0.14 & 0.77 \\
\hline LNAPL-water & 0.039 & 7.77 & 0.10 & 0.91 & 18.21 & 2.71 & 0.043 & 0.90 \\
\hline Air-LNAPL ${ }^{2)}$ & 0.043 & 7.37 & 0.10 & & 16.67 & 4.15 & 0.043 & \\
\hline Scaled parameters ${ }^{3)}$ & 0.021 & 7.51 & 0.00 & 0.90 & 38.04 & 3.79 & 0.00 & 0.85 \\
\hline
\end{tabular}

1) $R$ is a correlation coefficient

2) The $S$ - $p$ relations are obtained with scaling factor method

3) Scaled $S-p$ relation is obtained among air-water, LNAPL-water and air-LNAPL systems

tions including the type of sand used, the porosity of the sand samples, pore water, test equipments. The capillary pressure head in LNAPL-water system at a given water saturation is slightly lower compared to that in air-water system. This tendency is consistent with the results by Parker (1989) and Sharma and Mostafa (2003).

To obtain the residual water saturation, two methods can be utilized. One is to define residual water saturation, more or less, objectively by extrapolation using measured data, and the other is to determine residual water saturation at some arbitrarily large capillary pressure (Corey, 1994). To define the residual water saturation with extrapolation method, it should be noted that this procedure can become quite elaborate when only a small portion of the soil-water retention curve is measured (van Genuchten, 1980). In this paper, measured $S-p$ relation data was fitted to VG and BC models respectively with modified Gauss-Newton method (Hartley, 1961). The fitted $S-p$ relations with VG and BC models are also shown in Fig. 11. Fitting parameters of $\mathrm{VG}$ and $\mathrm{BC}$ models are shown in Table 2.

Figure 11 also indicate that the $S$ - $p$ relation of wetting process is much different to that of drainage process, and the hysteresis is clearly observed, as reported in previous researches. This is because LNAPL, which has infiltrated into the pore, is trapped with a degree of saturation even when the water infiltrated again.

\section{Prediction of S-p Relation in Air-LNAPL System}

The electrical conductivity probe can not be utilized in air-LNAPL system for measuring $S$ - $p$ relation because there is no pore water with some electrical conductivity in the system. For a rigid porous medium it is only necessary to measure $S$ - $p$ relations for two of the three two-phase systems (e.g., air-water, air-organic or organic-water). $S-p$ relations for the third system may then be predicted (Lenhard and Parker, 1987b). In the scaling procedure proposed by Parker et al. (1987), $S$ - $p$ relations of twophase air-water, air-organic and organic-water systems are adjusted to obtain a unique scaled function for a given porous medium after applying a linear transformation to capillary heads such that:

$$
\begin{aligned}
& S_{\mathrm{we}}\left(\beta_{\mathrm{aw}} h_{\mathrm{aw}}\right)=S^{*}\left(h^{*}\right) \\
& S_{\mathrm{we}}\left(\beta_{\mathrm{ow}} h_{\mathrm{ow}}\right)=S^{*}\left(h^{*}\right) \\
& S_{\mathrm{oe}}\left(\beta_{\mathrm{ao}} h_{\mathrm{ao}}\right)=S^{*}\left(h^{*}\right)
\end{aligned}
$$

where $a, o$, and $w$ refer to air, organic substance, e.g., LNAPL, and water phases respectively; $S_{\mathrm{je}}=\left(S_{\mathrm{j}}-S_{\mathrm{jr}}\right) /$ $\left(1-S_{\mathrm{jr}}\right)$ is the effective saturation of wetting phase $j$; $S_{\mathrm{jr}}$ is the irreducible saturation of wetting phase $j$; $\beta_{\mathrm{ij}}$ is a fluid pair-dependent scaling factor; and $S^{*}\left(h^{*}\right)$ is a scaled function between the saturation of wetting phase and capillary pressure head. At a given saturation of wetting phase, Eq. (13) indicates that:

$$
\beta_{\text {aw }} h_{\text {aw }}=\beta_{\text {ow }} h_{\text {ow }}=\beta_{\text {ao }} h_{\text {ao }}
$$

For a rigid porous medium, scaling coefficients are related by Lenhard and Parker (1987b):

$$
\frac{1}{\beta_{\text {ow }}}+\frac{1}{\beta_{\text {ao }}}=\frac{1}{\beta_{\text {aw }}}
$$

Accordingly, if $\beta_{\mathrm{a}}$ is known, at a given saturation of wetting phase, $h_{\mathrm{ao}}$ can be obtained depending on Eq. (14), 


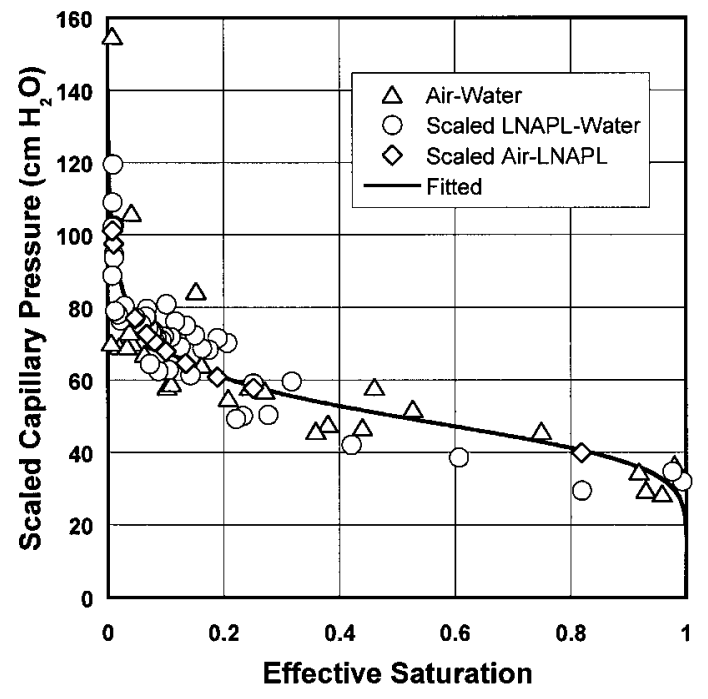

(a)

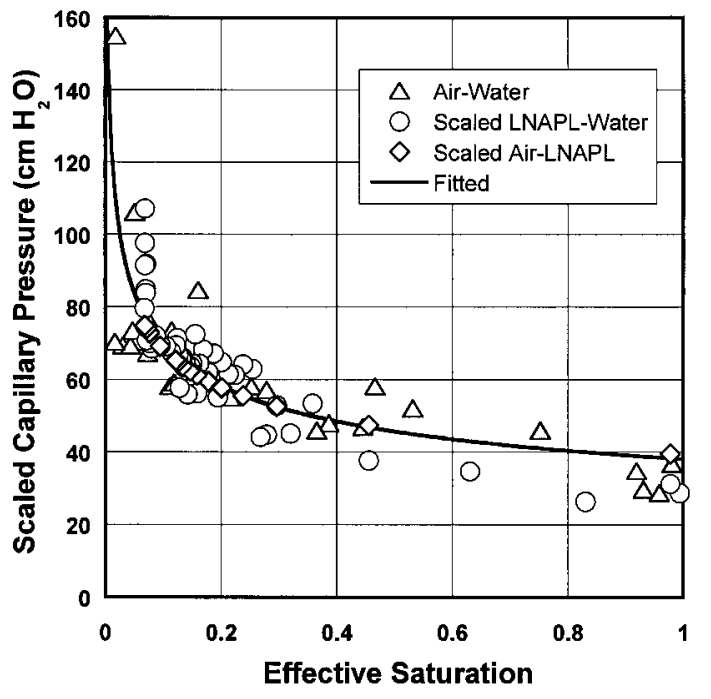

(b)

Fig. 12. Scaled effective saturation-capillary pressure relations in Toyoura sand: (a) VG model $\left(\beta_{\mathrm{ow}}=1.942, \beta_{\mathrm{ao}}=2.062\right)$ and (b) $\mathrm{BC}$ model $\left(\beta_{\mathrm{ow}}=\right.$ 1.739, $\beta_{\text {ao }}=2.352$ )

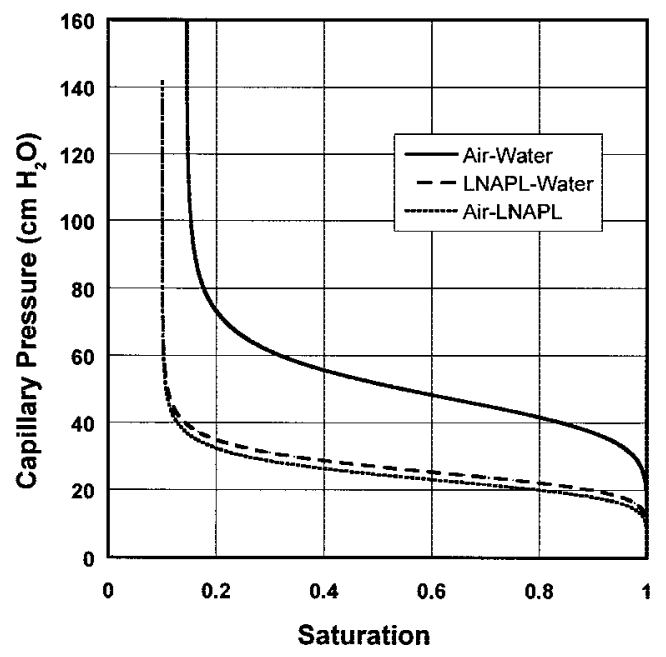

(a)

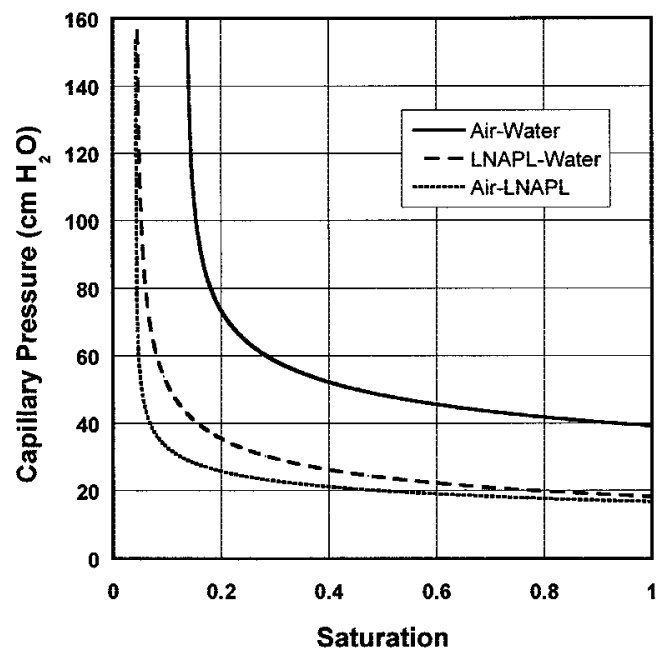

(b)

Fig. 13. $S$ - $p$ relations in Toyoura sand fitted with: (a) VG model and (b) BC model

and $S$ - $p$ relationship in air-LNAPL system can be obtained. The procedure for predicting $S$ - $p$ relationship in air-LNAPL system is described as follows:

(1) Taking the air-water system as a reference fluid pair system, and setting $\beta_{\mathrm{aw}}=1.0$;

(2) Fitting $S-p$ relationship data in the air-water system to obtain $S^{*}\left(h^{*}\right)$;

(3) Obtaining $\beta_{\text {ow }}$ depending on Eq. (13b) and $\beta_{\text {ao }}$ depending on Eq. (15);

(4) calculating $S_{\mathrm{oe}}$ and $h_{\mathrm{ao}}$ depending on Eq. (13c). where $S_{\mathrm{oe}}=\left(S_{\mathrm{o}}-S_{\mathrm{or}}\right) /\left(1-S_{\mathrm{or}}\right)$, and $S_{\mathrm{or}}$ is the residual saturation of wetting phase (LNAPL) in air-LNAPL system.

In the air-water and the LNAPL-water systems, water is a wetting phase fluid; both air and LNAPL are nonwetting fluids. Compared to air, LNAPL is a wetting phase fluid in air-LNAPL system. The residual saturation of wetting fluid is assumed to be a constant for all two-phase systems according to Lenhard and Parker (1987b), which indicates that the residual saturation of wetting fluid, LNAPL in air-LNAPL system is assumed to be 0.10 , which is the residual saturation of water fitted with test data, in LNAPL-water system. Furthermore, the relation among the scaling coefficient (Eq. (15)) is of a theory. In this paper, the $S$ - $p$ relation in the air-LNAPL can not be obtained experimentally. Thus Eq. (15) is utilized to estimate the $S-p$ relation in the air-LNAPL system.

The VG and BC parameters of predicted $S-p$ relation in the air-LNAPL system are shown in Table 2 . The scaled effective saturation-capillary pressure relations are shown in Fig. 12, and all the fitted $S$ - $p$ relations in air-water, airLNAPL and LNAPL-water systems are shown in Fig. 13. It can also be found that descending sequence of capillary pressure head at a given saturation of wetting phase is in 


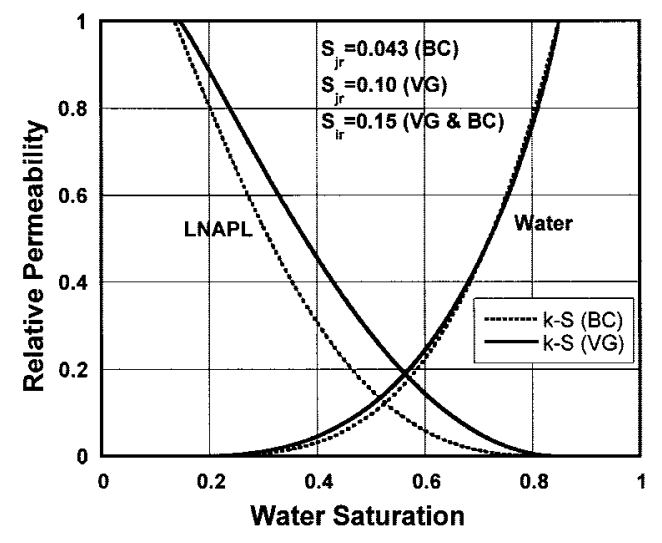

(a)

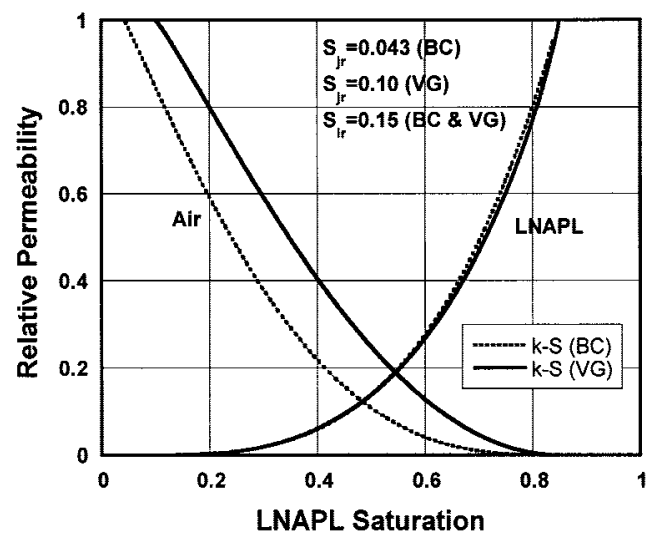

(b)

Fig. 14. Estimated $k-S$ relations in Toyoura sand: (a) LNAPL-water system and (b) Air-LNAPL system

the order of air-water, LNAPL-water and air-LNAPL systems. This tendency is also consistent with the results shown by Parker (1989) and Sharma and Mostafa (2003).

In air-water and LNAPL-water systems, the entry pressure and displacement pressure of air-water system are larger than that of LNAPL-water system. This result suggests that, in their corresponding aqueous two phase systems, LNAPL is easier to displace water than air during the drainage process. In air-water and air-LNAPL systems, the entry pressure and displacement pressure of air-water system are larger than that of air-LNAPL system. This also suggests that LNAPL is easier to be desaturated than water in their corresponding gases two phase systems.

\section{Relative Permeability-Saturation Relations in LNAPL- Water and Air-LNAPL Systems}

Based on Eqs. (8) to (11), a relation between the relative permeability and the saturation of wetting phase can be estimated based on the $S-p$ relations obtained. The effective saturation of wetting phase $j$ here is described as;

$$
S_{\mathrm{je}}=\frac{S_{\mathrm{j}}-S_{\mathrm{jr}}}{1-S_{\mathrm{jr}}-S_{\mathrm{ir}}}
$$

where, $S_{\mathrm{jr}}$ and $S_{\mathrm{ir}}$ are the irreducible saturations of wetting phase $j$ and non-wetting phase $i$, respectively. $S_{\mathrm{jr}}$ can be obtained with extrapolation method and a good estimate of $S_{\text {ir }}$ for homogeneous and isotropic media is 0.15 (Corey, 1994).

The relative permeability-saturation relations ( $k-S$ relations) estimated for air-LNAPL and LNAPL-water systems are shown in Fig. 14. The values of relative permeability of nonwetting fluids (e.g., air in air-LNAPL system and LNAPL in LNAL-water system) estimated with $\mathrm{BC}$ model is slightly smaller than that estimated with VG model at a given saturation of wetting phase (e.g., water in air-water system and LNAPL in air-LNAPL system). On the other hand, the relative permeability of wetting fluid estimated with $\mathrm{BC}$ model is nearly the same as that estimated with VG model.

The Brooks-Corey model is applied in conjunction with the Burdine theorem (1953), in which, pores are represented with a group of parallel capillary tubes with different radii. The van Genuchten model is applied in conjunction with the more complicated pore geometry model of Mualem (1976). Due to the differences in parameters between Burdine model and Mualem model, the relative permeability of $\mathrm{BC}$ model is always smaller than that of VG model (Oostrom and Lenhard, 1998).

With regard to the relative permeability, few literatures are found on the differences between the relative permeability measured by way of test and that predicted by way of BC and VG models in porous media in which LNAPL exists. However some simplified researches have been made. In a fracture network, it was found that, both the VG and $\mathrm{BC}$ models generally underestimate relative permeability values, while the $\mathrm{BC}$ model gives better results than the VG model (Liu and Bodvarsson, 2001). In porous media, the predictions based on the $\mathrm{BC}$ model also agreed better with experimental observations than the predictions based the VG model (Schroth et al., 1998). It can also be found that the BC model used by engineers successfully predicts the relative permeability curves (Dana and Skoczylas, 1999). In addition, modified prediction methods for the relative permeability including the effects of wettability, contact angle of soil particles, and DNAPL entrapment under water-DNAPL two-phase condition have been also developed (Demond and Roberts, 1987; Morrow et al., 1985). However, further research is required to develop the model to determine the relative permeability of NAPL and evaluate its validity.

The differences of $k-S$ relations between air-water, LNAPL-water, and air-LNAPL systems are shown in Fig. 15. It indicates that the relative permeability of air is larger than that of LNAPL in their corresponding aqueous two phase systems. It also suggests that the relative permeability of the LNAPL is slightly larger than that of water in their corresponding gaseous two phase systems. Since these relative permeability values are estimated based on Eqs. (8) to (11), these differences are due to the shapes of $S-p$ relation, which includes the effects of many factors (dynamic interfacial tension, viscosity, and so on) on the multiphase infiltration 


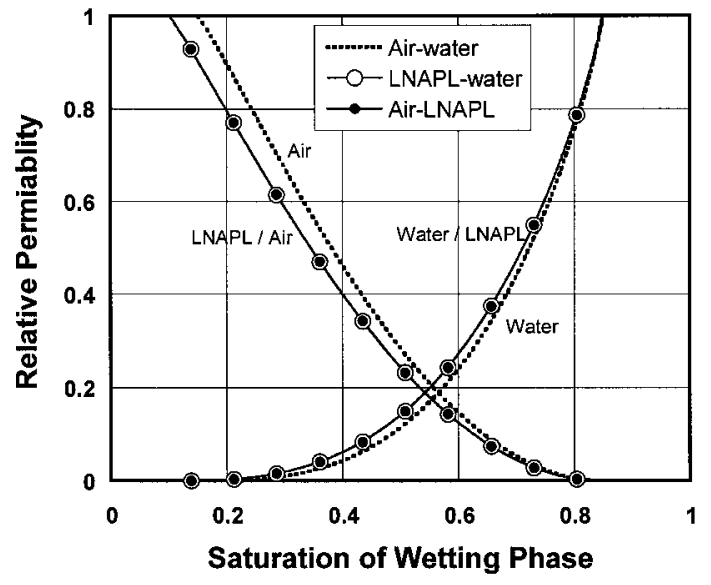

(a)

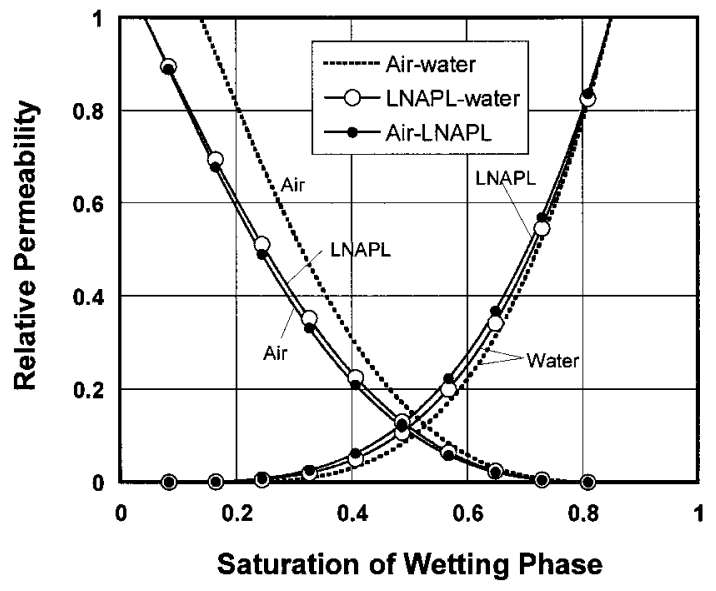

(b)

Fig. 15. Differences in $k-S$ relations between air-water, LNAPL-water, and air-LNAPL systems estimated using: (a) VG model and (b) BC model

properties, obtained for the corresponding phase system.

\section{CONCLUSIONS}

In this paper, the experimental system for the measurement of migration characteristics of LNAPL (i.e., $S-p$ relation) in LNAPL-water system in the porous medium is described. Using this system, $S-p$ relation in the LNAPL-water system is characterized, and that in the air-LNAPL system is determined by the scaling factor method. The main results obtained are as follows:

(1) A simple experiment device system with electrical conductivity probes and tensiometers is effective for measuring $S$ - $p$ relation in LNAPL-water system.

(2) Relations between water saturation and output voltage across a resistance attached to a calibrated electrical conductivity probe were determined in the laboratory calibration tests. The output voltagewater saturation relation in LNAPL-water system is slightly different from that in air-water system. In air-water system, the curvature of the relation is slightly larger.

(3) The method for modifying a hydrophilic tensiometer to a hydrophobic one is proved to be effective for measuring the pore LNAPL pressure even in the case of a highly-viscous LNAPL such as paraffin liquid used in this study.

(4) A method to separate sand particle, LNAPL and water from the sand sample collected from the column test was designed and proved to be effective in the laboratory experiments.

(5) At a given water saturation, the measured capillary pressure in LNAPL-water system is slightly smaller than that in air-water system. In reference to scaling factors, $S-p$ relation in air-LNAPL system is obtained. The descending sequence of capillary pressure is in the order of air-water, LNAPL-water and air-LNAPL systems. This is consistent with the results by Kamon et al. (2003) and Sharma and Mostafa (2003).
(6) The $k-S$ relation is obtained with parameters fitted with VG and BC model in LNAPL-water and airLNAPL systems. The relative permeability of LNAPL obtained using BC model is slightly smaller than that obtained using VG model, and the relative permeability of water obtained using $\mathrm{BC}$ model is similar to that obtained using VG model in both LNAPL-water and air-LNAPL systems.

(7) The entry pressure and displacement pressure of airwater system are larger than that of LNAPL-water system. It suggests that, in their corresponding aqueous two phase systems, LNAPL is easier to displace water than air at the beginning of drainage process. The entry pressure and displacement pressure of air-water system are larger than that of air-LNAPL system. It also suggests that LNAPL is easier to be desaturated than water in their corresponding gaseous two phase systems.

(8) The relative permeability of air is larger than that of LNAPL in their corresponding aqueous two phase systems. In addition, the relative permeability of the LNAPL is slightly larger than that of water in their corresponding gaseous two phase systems.

\section{ACKNOWLEDGEMENTS}

The authors would like to acknowledge Dr. Huyuan Zhang (Former JSPS Research Fellow, Graduate School of Global Environmental Studies, Kyoto University, Japan) for his support in the laboratory test.

\section{NOTATION}

$A$ : fitted parameter

$B$ : fitted parameter

$g$ : gravitational acceleration

$h_{\text {cij }}$ : capillary pressure head between fluid pair of $i$ and $j$

$h_{\mathrm{d}}$ : displacement pressure head

$h_{\text {aw: }}$ : capillary pressure head in air-water system

$h_{\text {ow }}$ : capillary pressure head in LNAPL-water system

$h_{\mathrm{ao}}$ : capillary pressure head in air-LNAPL system 
$k$ : permeability of a medium

$k_{\mathrm{ei}}: \quad$ effective permeability of $i$ phase in multiphase flow case

$k_{\mathrm{ri}}:$ relative permeability of nonwetting phase $i$

$k_{\mathrm{rj}}$ : relative permeability of wetting phase $j$

$m$ : parameter fitted with van Genuchten model

$n$ : parameter fitted with van Genuchten model

$p_{\mathrm{e}}$ : entry pressure

$p_{\mathrm{d}}:$ displacement pressure

$P_{\mathrm{i}}$ : nonwetting phase pressure

$P_{\mathrm{j}}$ : $\quad$ wetting phase pressure

$P_{\mathrm{ij}}$ : capillary pressure between phases pair of $i$ and $j$

$P_{\text {aw }}:$ capillary pressure between air and water

$P_{\mathrm{ow}}:$ capillary pressure between LNAPL and water

$P_{\mathrm{ao}}$ : capillary pressure between air and LNAPL

$R_{\mathrm{ij}}^{*}$ : harmonic mean radius of curvature of the interface between phase $i$ and phase $j$

$R^{*}$ : harmonic mean radius of curvature of the interface

$R:$ output voltage

$R_{0}$ : reference output voltage

$S_{\mathrm{w}}$ : water saturation

$S_{\text {we }}$ : effective water saturation

$S_{\mathrm{oe}}$ : effective saturation of LNAPL

$S_{\mathrm{j}}$ : $\quad$ saturation of wetting phase $j$

$S_{\mathrm{r}}: \quad$ residual saturation

$S_{\mathrm{je}}: \quad$ effective saturation of wetting phase

$S_{\mathrm{ir}}: \quad$ residual saturation of nonwetting phase $i$

$S_{\mathrm{j} \mathrm{r}}: \quad$ residual saturation of wetting phase $j$

$S^{*}\left(h^{*}\right)$ : scaled saturation-capillary head function

$v_{\mathrm{i}}$ : velocity of fluid in a porous medium

$W_{\text {total }}:$ total weight of sample including sand, LNAPL and water

$W_{\mathrm{S}}$ : weight of sand

$W_{\mathrm{L}}$ : weight of LNAPL

$\alpha: \quad$ parameter fitted with van Genuchten (VG) model

$\beta_{\text {aw }}$ : air-water fluid pair-dependent scaling factor

$\beta_{\text {ow }}$ : LNAPL-water fluid pair-dependent scaling factor

$\beta_{\text {ao }}$ : air-LNAPL fluid pair-dependent scaling factor

$\rho_{\mathrm{w}}$ : density of water

$\lambda$ : parameter fitted with Brooks and Corey (BC) model

$\varepsilon$ : parameter of VG model

$\mu_{\mathrm{i}}$ : viscosity of $i$ phase

$\sigma_{\mathrm{ij}}: \quad$ interfacial tension between phases $i$ and $j$

$\sigma_{\mathrm{aw}}$ : interfacial tension between air and water

$\sigma_{\text {ow }}:$ interfacial tension between LNAPL and water

$\sigma_{\mathrm{ao}}$ : interfacial tension between air and LNAPL

\section{REFERENCES}

1) Archie, G. E. (1942): The electrical resistivity log as an aid in determining some reservoir characteristics, Trans. A.I.M.E., 146, 54-61.

2) Archie, G. E. (1947): Electrical resistivity an aid in core-analysis interpretation, Bull. Am. Assoc. Petr. Geol., A.I.M.E., 31(2), 350-366.

3) Bear, J. (1979): Hydraulics of Groundwater, McGraw-Hill, New York, USA.

4) Brooks, R. H. and Corey, A. T. (1964): Hydraulic properties of porous media, Hydrology Paper, Colorado State University, Fort Collins, 3, 1-27.

5) Burdine, N. T. (1953): Relative permeability calculations from pore-size distribution data. Petr. Trans., Am. Inst. Mining Metall. Engng, 198, 71-77.

6) Busby, R. D., Lenhard, R. J. and Rolston, D. E. (1994): An investigation of saturation-capillary pressure relations in two-and-three fluid systems for several NAPLs in different porous media, Ground Water, 33, 570-578.

7) Chapra, S. C. and Canale, R. P. (1998): Numerical Methods for Engineers with Programming and Software Applications, 3rd ed., 508-511.

8) Corey, A. T. (1994): Mechanics of Immiscible Fluids in Porous Media, Water Resources Publications, 41-43.
9) Dana, E. and Skoczylas, F. (1999): Gas relative permeability and pore structure of sandstones, Int. J. Rock Mech. Min. Sci., 36, 613-625.

10) Demond, A. H. and Roberts, P. V. (1987): An examination of relative permeability relations for two-phase flow in porous media, Water Resour. Bull., 23, 617-628.

11) Dullien, F. A. L. (1992): Porous Media: Fluid Transport and Pore Structure, 2nd ed., Academic Press, California, USA.

12) Endo, K. (2002): Characteristics of DNAPL migration and assessment of DNAPL distribution in a contaminated site, $P h D$ Dissertation, Kyoto University, Japan (in Japanese).

13) Gardner, W. and Kirkham, D. (1951): Determination of soil moisture by neutron scattering, Soil Sci., 73, 391-401.

14) Hartley, H. O. (1961): The modified Gauss-Newton method for the fitting of non-linear regression functions by least squares, Technometrics, 3(2), 269-280.

15) Helmig, R. (1997): Multiphase Flow and Transport Processes in the Subsurface, Springer, Berlin.

16) Host-Madsen, J. and Jensen, K. H. (1992): Laboratory and numerical investigation of immiscible multiphase flow in soil, $J$. Hydrol., 135, 13-52.

17) Huh, C. and Mason, S. G. (1975): A rigorous theory of ring tensiometry colloid polymer, Science, 253, 566-580.

18) Iitani, K. and Masuda, H. (1975): Automatic process of powder, The Nikkan Kogyo Shimbun Ltd., Japan (in Japanese).

19) Illangasekare, T. H., Ramsey, J. L., Jensen, K. H. and Butts, M. B. (1995a): Experimental study of movement and distribution of dense organic contaminants in heterogeneous aquifers, J. Cont. Hydr., 20, 1-25.

20) Illangasekare, T. H., Armbruster III, E. J. and Yates, D. N. (1995b): Non aqueous phase fluids in heterogeneous aquifers, Experimental Study, J. Environ. Eng., 571-579.

21) Kamon M., Endo, K. and Katsumi, T. (2003): Measuring the $k-S-p$ relations on DNAPLs migration, Eng. Geol., 70, 351-363.

22) Kechavarzi, C., Soga, K. and Wiart, P. (2000): Multispectral image analysis method to determine dynamic fluid saturation distribution in two-dimensional three-fluid phase flow laboratory experiments, J. Cont. Hydr., 46, 265-293.

23) Lenhard, R. J. and Parker, J. C. (1987a): A model for hysteretic constitutive relations governing multiphase flow: 2 . Permeabilitysaturation relations, Water Resour. Res., 23, 2197-2206.

24) Lenhard, R. J. and Parker, J. C. (1987b): Measurement and prediction of saturation-pressure relationships in three-phase porous media systems, J. Cont. Hydr., 1, 407-424.

25) Lenhard, R. J. and Parker, J. C. (1988): Experimental validation of the theory of extending two-phase saturation-pressure relations to three-fluid phase systems for monotonic drainage paths, Water Resour. Res., 24, 373-380.

26) Lenhard, R. J., Johnson, T. G. and Parker, J. C. (1993): Experimental observation of non-aqueous-phase liquid subsurface movement, J. Cont. Hydr., 12, 79-101.

27) Liu, H. H. and Bodvarsson, G. S. (2001): Constitutive relations for unsaturated flow in a fracture network, J. Hydr., 252, 116-125.

28) Morrow, N. R., Chatzis, I. and Lim, H. (1985): Relative permeabilities at reduce residual saturations, J. Can. Pet. Technol., 62-69.

29) Mualem, Y. (1976): A new model for predicting the hydraulic conductivity of unsaturated porous mediua, Water Resour. Res., 12, 513-522.

30) Oostrom, M. and Lenhard, R. J. (1998): Comparison of relative permeability-saturation-pressure parametric models for infiltration and redistribution of a light nonaqueous-phase liquid in sandy porous media, Adv. Water Resour., 21, 145-157.

31) Parker, J. C., Lenhard, R. J. and Kuppusamy, T. (1987): A parametric model for constitutive properties governing multiphase flow in porous media, Water Resour. Res., 23, 618-624.

32) Parker, J. C. (1989): Multiphase flow and transport in porous media, Rev. Geophys., 27(3), 311-328.

33) Reginato, R. J. and van Bavel, C. H. M. (1964): Soil water measurement with gamma attenuation, Soil Sci. Soc. Am. Proc., 28, 721-724.

34) Schroth, M. H., Istok, J. D., Selker, J. S., Oostrom, M. and 
White, M. D. (1998): Multifluid flow in bedded porous media: laboratory experiments and numerical simulations, $A d v$. Water Resour., 22, 169-183.

35) Sharma, R. S. (2000): Keynote paper: migration of non-aqueous phase liquids in subsurface, Int. Conf. Geo-environment, Muscat, Sultanate of Oman, Balkema, Rotterdam, The Netherlands, 2, 571-583.

36) Sharma, R. S. and Mostafa H. A. (2003): An experimental investigation of LNAPL migration in an unsaturated/saturated sand, Eng. Geol., 70, 305-313.
37) Tidwell, V. C. and Glass, R. J. (1994): X-ray and visible light transmission for laboratory measurements of two-dimensional saturation fields in thin-slab systems, Water Resour. Res., 30, 2873-2882.

38) Topp, G. C., Davis, J. L. and Annan, A. P. (1980): Electromagnetic determination of soil water content: measurements in coaxial transmission lines, Water Resour. Res., 16, 574-582.

39) van Genuchten, M. TH. (1980): A closed-form equation for predicting the hydraulic conductivity of unsaturated soils, Soil Sci. Soc., AM. J., 44, 892-898. 